\section{Azathioprine and diffuse alveolar haemorrhage: the pharmacogenetics of thiopurine methyltransferase}

\author{
D. Perri*,\#, , D.E.C. Cole ${ }^{+, \varsigma_{,},}$, O. Friedman ${ }^{+}$, E. Piliotis ${ }^{+}$, S. Mintz ${ }^{+}$and N.K.J. Adhikari**
}

ABSTRACT: Current guidelines support the use of corticosteroids and azathioprine as one possible treatment strategy for idiopathic pulmonary fibrosis (IPF). However, some patients with genetic polymorphisms of thiopurine methyltransferase (TPMT) are at risk of severe azathioprine myelotoxicity.

The current authors present the case of an 85-yr-old Caucasian male with IPF who developed diffuse alveolar haemorrhage as a complication of azathioprine-induced myelosuppression.

Leukocyte genetic TPMT testing revealed that the patient had homozygous polymorphisms associated with the absence of TPMT activity and severe azathioprine-induced myelotoxicity.

Thiopurine methyltransferase deficiency should be considered in patients who develop leukopenia early in treatment with azathiopurine, or who present with severe marrow suppression at usual doses. For centres with equipped laboratories, a dosing suggestion is provided based on thiopurine methyltransferase testing. Even with screening strategies, frequent monitoring of complete blood count and liver biochemistry should remain the mainstay of surveillance for azathioprine toxicity.

KEYWORDS: Azathioprine, diffuse alveolar haemorrhage, myelotoxicty, pharmacogenetics, polymorphism, thiopurine methyltransferase

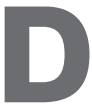
espite the lack of data to support the use of immunosuppressive drugs for idiopathic pulmonary fibrosis (IPF), clinicians should not necessarily dismiss them as ineffective [1, 2]. In fact, the American Thoracic Society/European Respiratory Society consensus statement suggests moderate doses of steroids combined with either azathioprine or cyclophosphamide [3]. Azathioprine is a pro-drug of 6mercaptopurine (6-MP). It is used as an immunosuppressant for solid organ and haematological transplants, as well as a steroid-sparing agent for a variety of immune-mediated diseases. Its use is limited by both its slow onset of action (34 months) and its toxicity (sometimes occurring before any anti-inflammatory effect), which includes hepatitis, bone marrow suppression, infection and malignancy.

\section{CASE REPORT}

An 85-yr-old Caucasian male was admitted to hospital with fatigue and increasing dyspnoea of

For editorial comments see page $\mathbf{8 2 1}$.
2 weeks' duration. He had been diagnosed with IPF 4 yrs previously. His other past medical history included transurethral resection of bladder cancer, hypertension and chronic kidney disease (creatinine $150 \mu \mathrm{mol} \cdot \mathrm{L}^{-1}$, normal levels 44-106 $\left.\mu \mathrm{mol} \cdot \mathrm{L}^{-1}\right)$. His dyspnoea had progressed over the months prior to hospitalisation, limiting his mobility and necessitating supplemental home oxygen therapy. Due to the patient's worsening clinical status and deteriorating pulmonary function tests, he was prescribed azathioprine $100 \mathrm{mg}$ daily and prednisone $40 \mathrm{mg}$ daily starting 2 months prior to hospital admission. His other medications included beclomethasone inhalations, nadolol and hydrochlorothiazide. Shortly after the patient was admitted to hospital, he developed acute respiratory failure. Significant findings on physical examination included paradoxical breathing, tachypnoea (36 breaths $\left.\cdot \mathrm{min}^{-1}\right)$, a high oxygen requirement (oxygen saturation $92 \%$ on oxygen $15 \mathrm{~L} \cdot \mathrm{min}^{-1}$ by a nonrebreather mask) and bilateral anterolateral crackles on chest auscultation. A chest

\section{AFFILIATIONS}

*Depts of Pharmaceutical Sciences, ${ }^{+}$Medicine,

§Laboratory Medicine and

Pathobiology,

${ }^{f}$ Pediatrics (Genetics), and

** Interdepartmental Division of

Critical Care Medicine, University of

Toronto, Toronto,

\#Dept of Medicine, McMaster

University, and

'Centre for Evaluation of Medicines,

St. Joseph's Healthcare, Hamilton,

ON, Canada.

\section{CORRESPONDENCE}

N.K.J. Adhikari

Dept of Critical Care Medicine

Room D1.08

Sunnybrook Health Sciences Centre

2075 Bayview Avenue

Toronto

ON

M4N 3M5

Canada

Fax: 14164804999

E-mail: neill.adhikari@

sunnybrook.ca

Received:

March 062007

Accepted after revision:

July 062007

STATEMENT OF INTEREST

None declared. 
radiograph revealed diffuse airspace disease with basal honeycombing. The patient was intubated and admitted to the intensive care unit (ICU) for mechanical ventilation. His complete blood count was as follows: haemoglobin $65 \mathrm{~g} \cdot \mathrm{L}^{-1}$ (normal level 130-180 $\mathrm{g} \cdot \mathrm{L}^{-1}$ ), with a high normal mean corpuscular volume; reticulocyte count $1 \times 10^{9} \cdot \mathrm{L}^{-1}$ (normal level $10-75 \times 10^{9} \cdot \mathrm{L}^{-1}$ ); platelet count $17 \times 10^{9} \cdot \mathrm{L}^{-1}$ (normal level $\left.150-400 \times 10^{9} \cdot \mathrm{L}^{-1}\right)$; and white blood cell count $0.4 \times 10^{6} \cdot \mathrm{L}^{-1}$ (normal level $4.5-11 \times 10^{6} \cdot \mathrm{L}^{-1}$ ), with $0.1 \times 10^{6} \cdot \mathrm{L}^{-1}$ neutrophils and $0.2 \times 10^{6} \cdot \mathrm{L}^{-1}$ lymphocytes. Electrolytes, liver function tests and creatinine kinase were unremarkable; serum creatinine was elevated but remained near baseline level. Fibreoptic bronchoscopy showed diffuse bleeding, and sequential bronchoalveolar lavages (BALs) were bloody. The diagnoses upon admission were azathioprine-induced pancytopaenia and diffuse alveolar haemorrhage.

The patient developed a fever shortly after ICU admission and was treated empirically for febrile neutropaenia with broadspectrum antibiotics and filgrastim. Cultures of BAL fluid and blood grew Staphylococcus aureus; BAL was negative for atypical cells, viruses, fungal elements and Pneumocystis jiroveci. Transoesophageal echocardiography showed no evidence of endocarditis. A sample for thiopurine methyltransferase (TPMT) genetic testing was taken, and the patient received platelets and packed red blood cells. Due to nonresolving respiratory failure, and in accordance with the patient's previously expressed wishes, life support was withdrawn on the seventh hospital day. The family declined permission for a post-mortem examination. TPMT activity was not measured by the current authors' laboratory, but molecular genetic testing reported 2 weeks later revealed the patient to be homozygous for the TPMT*3A (460G $>$ A and 719A $>\mathrm{G}$ ) mutation and, thus likely to have low or no TPMT activity. The trigger for the patient's diffuse alveolar haemorrhage was not pathologically established, but the available results suggested the combination of infection and azathioprineinduced thrombocytopaenia.

\section{DISCUSSION}

As shown in figure 1, azathioprine is converted to 6-MP in vivo, where it is subsequently metabolised to either 6thioguanine (6-TG) nucleotides by the enzyme inosine- $5^{\prime}$ monophosphate dehydrogenase (IMPDH), or to 6-methylmecaptopurine ribonucleotides by the enzyme TPMT [4, 5]. 6-TG is a cytotoxic metabolite associated with marrow suppression. TPMT also converts 6-MP to 6-methyl-mercaptopurine, which is associated with hepatotoxicity.

The activity of the enzyme TPMT has been shown to be trimodal, with $90 \%$ of people having high activity, $10 \%$ intermediate, and $0.3 \%$ low or no activity $[6,7]$. In those with reduced activity, the metabolism of 6-MP is shunted to the IMPDH pathway, leading to accumulation of myelotoxic 6-TG nucleotides. The current authors are unaware of direct experimental evidence that explains why the xanthine oxidase pathway does not accommodate the excess 6-MP. TPMT activity is related to $\sim 20$ different single nucleotide polymorphisms [8]. Three key mutations, however, account for $>90 \%$ of all clinically significant TPMT mutations and $80-95 \%$ of low or intermediate metabolisers found in Caucasian, African-American and Asian sub-populations. The common mutant haplotypes are $\mathrm{TPMT}^{*} 2 \quad(238 \mathrm{G}>\mathrm{C}$ predicting Ala80Pro), the double heterozygote TPMT*3A (460G $>$ A and 719A $>$ G predicting Ala154Tyr and Tyr240Cys) and TPMT*3C (719A $>\mathrm{G}$ alone) $[6,9]$. TPMT ${ }^{*} \mathrm{~A}$ is the most prevalent deleterious allele among Caucasians, while TPMT*3C is the most prevalent in African and South-East Asian populations. Patients with intermediate activity are usually heterozygous (i.e. have one mutated and one "wild-type" chromosome), while those with low or absent TPMT activity are almost always homozygous for TPMT mutations (i.e. both chromosomes are mutated).

TPMT phenotype correlates with 6-TG metabolite levels, thought to result in bone marrow toxicity $[10,11]$, and TPMT status may predict the duration from initiation of therapy to myelosuppression. In a series of rheumatoid arthritis patients taking azathioprine, five out of six subjects heterozygous for a mutant allele had therapy discontinued within 1 month due to low leukocyte counts [12]. In a study of Crohn's disease patients receiving azathioprine, cytopaenia manifested within 2 months for homozygotes, within 16 months for heterozygotes, and as late as 87 months for those with no mutated TPMT alleles [13]. Similar studies in patients taking azathioprine for lung disease have yet to be carried out.

The use of other myelosuppressive drugs, or the presence of concurrent infections, could explain the lack of specificity in TPMT testing studies. Furthermore, concurrent use of allopurinol, a xanthine oxidase inhibitor, may cause a toxic shift in the metabolism of thiopurines. Preliminary studies looking at another enzyme involved in azathioprine metabolism, inosine triphosphate pyrophosphatase (ITPA), show that myelosuppresion may also be related to certain relatively rare ITPA gene polymorphisms [14, 15]. In summary, while factors other than TPMT status are likely to be involved in azathioprine toxicity, the majority of evidence suggests that TPMT deficiency is a

Liver Erythrocyte

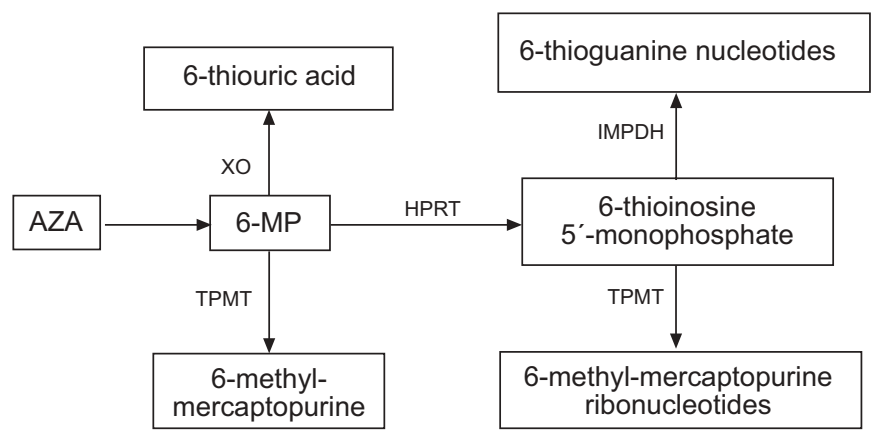

FIGURE 1. A simplified schematic diagram showing the major pathways of mercaptopurine metabolism. Azathioprine (AZA) is converted to 6-mercaptopurine (6-MP) in the liver via a glutathione-dependent process accelerated by glutathioneS-transferase. 6-MP undergoes further metabolism by xanthine oxidase $(\mathrm{XO})$ and thiopurine methyltransferase (TPMT). 6-MP is also metabolised by hypoxanthine guanine phosphoribosyltransferse (HPRT) within the red blood cells and is subsequently converted by TPMT to 6-methyl-mercaptopurine ribonucleotides or by inosine-5'- monophosphate dehydrogenase (IMPDH) to 6-thioguanine nucleotides. Deficiency of TPMT activity leads to accumulation of 6-thioguanine nucleotides, which may cause bone marrow toxicity. 


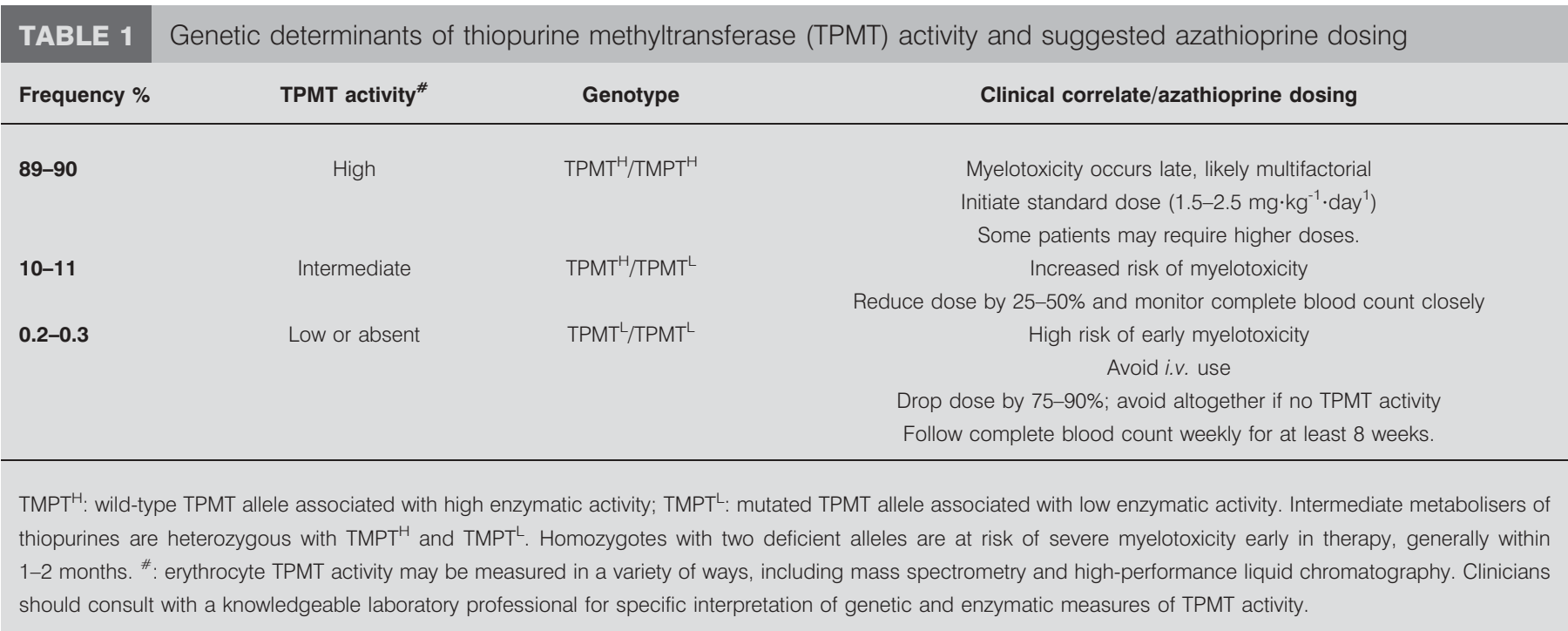

significant independent risk factor for myelotoxicity, especially early in the course of treatment.

A recent study in renal transplant patients initiated on $1.5 \mathrm{mg} \cdot \mathrm{kg}^{-1} \cdot \mathrm{day}^{-1}$ of azathioprine found that twice the proportion of patients with a heterozygous TPMT mutation required dose reductions due to leukopenia than patients with homozygous wild-type TPMT [16]. Azathioprine dosing based on erythrocyte TPMT activity has been offered as a means to reduce the risk of cytopaenia [10, 17]. Although $>70 \%$ of myelosuppression related to azathioprine use is not associated with TPMT polymorphism [13, 18, 19], screening as few as 20 patients for TPMT deficiency can prevent one adverse event over 6 months of therapy [20]. Furthermore, pharmacoeconomic models and prospective studies have demonstrated that genotype or phenotype screening for TPMT polymorphisms is cost-effective in patients with rheumatological disorders [20], inflammatory bowel disease [21, 22], paediatric leukaemia [23] and autoimmune skin disease [24]. Other small prospective screening studies of TPMT activity have not adequately predicted toxicity in patients with inflammatory bowel disease $[25,26]$. Economic models are limited, as they are based only on literature estimates of the incidence of azathioprine toxicity. However, large-scale prospective screening studies are unavailable, and with mixed results in the small studies there is ongoing debate regarding the benefit of routine TPMT screening. The British Society of Gastroenterology guidelines state that TPMT measurement cannot yet be recommended as a prerequisite to therapy with azathioprine [27], a view shared by the American College of Gastroenterology [28]. The British Association of Dermatologists, however, suggests TPMT activity testing prior to initiating therapy with azathioprine [17]. The current authors are not aware of any pharmacoeconomic analyses or prospective screening studies of TPMT in patients with pulmonary diseases.

\section{Conclusion}

The patient was homozygous for the TPMT*3A mutation, thus putting him at greater risk for azathioprine myelotoxicity. The ensuing severe thrombocytopaenia is likely to have contributed to the diffuse alveolar haemorrhage. In this case, if the patient's TPMT status had been known in advance, it is possible that an alternative therapy may have been selected, especially given the lack of data supporting any particular pharmacological treatment strategy for IPF [29].

Since the utility and cost-effectiveness of thiopurine methyltransferase phenotype or genotype screening remains somewhat controversial, there are currently no national or international guidelines that recommend routine thiopurine methyltransferase testing prior to initiation of azathioprine for chest diseases. For those clinicians with access to thiopurine methyltransferase activity or genetic testing, table 1 summarises one possible clinical approach to the initiation of azathioprine. Where testing is unavailable, clinicians should be aware that one in 10 patients has a thiopurine methyltransferase polymorphism that may predispose them to azathioprine myelotoxicity. During the first 8 weeks of therapy, a minimum of weekly monitoring of the complete blood count and liver function tests should be considered, as this is the period of highest risk. If red blood cell thiopurine methyltransferase activity measurement is considered for patients on azathioprine presenting with pancytopaenia, blood samples should be drawn before the administration of red cell transfusions and should be interpreted based on the guidelines provided by the testing laboratory. Molecular genetic testing of leukocytes can be done irrespective of the administration of blood products.

\section{ACKNOWLEDGEMENTS}

The authors would like to thank B.Y.L. Wong and G. Koultchitski (Sunnybrook Health Sciences Centre, Toronto, ON, Canada) for performing thiopurine methyltransferase genotype testing (established by M. Reis, Sunnybrook Health Sciences Centre).

\section{REFERENCES}

1 Gross TJ, Hunninghake GW. Idiopathic pulmonary fibrosis. N Engl J Med 2001; 345: 517-525.

$2 \mathrm{du}$ Bois RM. Is idiopathic pulmonary fibrosis now treatable? Am J Respir Crit Care Med 2005; 171: 939-940. 
3 American Thoracic Society. Idiopathic pulmonary fibrosis: diagnosis and treatment. International consensus statement. American Thoracic Society (ATS) and the European Respiratory Society (ERS). Am J Respir Crit Care Med 2000; 161: 646-664.

4 Lennard L. The clinical pharmacology of 6-mercaptopurine. Eur J Clin Pharmacol 1992; 43: 329-339.

5 McLeod HL, Krynetski EY, Relling MV, Evans WE. Genetic polymorphism of thiopurine methyltransferase and its clinical relevance for childhood acute lymphoblastic leukemia. Leukemia 2000; 14: 567-572.

6 Yates CR, Krynetski EY, Loennechen T, et al. Molecular diagnosis of thiopurine S-methyltransferase deficiency: genetic basis for azathioprine and mercaptopurine intolerance. Ann Intern Med 1997; 126: 608-614.

7 McLeod HL, Siva C. The thiopurine S-methyltransferase gene locus - implications for clinical pharmacogenomics. Pharmacogenomics 2002; 3: 89-98.

8 Schaeffeler E, Fischer C, Brockmeier D, et al. Comprehensive analysis of thiopurine S-methyltransferase phenotype-genotype correlation in a large population of German-Caucasians and identification of novel TPMT variants. Pharmacogenetics 2004; 14: 407-417.

9 Evans WE, Hon YY, Bomgaars L, et al. Preponderance of thiopurine S-methyltransferase deficiency and heterozygosity among patients intolerant to mercaptopurine and azathioprine. J Clin Oncol 2001; 19: 2293-2301.

10 Seidman EG. Clinical use and practical application of TPMT enzyme and 6-mercaptopurine metabolite monitoring in IBD. Rev Gastroenterol Disord 2003; 3: Suppl. 1, S30-S38.

11 Dervieux T, Matsutani M, Barry M, Siedman E. Drug metabolite monitoring of azathioprine/6-MP therapy: contribution of thiopurine methyltransferase gene polymorphisms: 69. Ther Drug Monit 2005; 27: 228.

12 Black AJ, McLeod HL, Capell HA, et al. Thiopurine methyltransferase genotype predicts therapy-limiting severe toxicity from azathioprine. Ann Int Med 1998; 129: 716-718.

13 Colombel JF, Ferrari N, Debuysere H, et al. Genotypic analysis of the thiopurine $S$-methyltransferase gene in patients with Crohn's disease and severe myelosuppression during azathioprine therapy. Gastroenterology 2000; 118: 1025-1030.

14 Zelinkova Z, Derijks LJ, Stokkers PC, et al. Inosine triphosphate pyrophosphatease gene mutations predicts myelosuppresion in IBD patients treated with azathioprine. Gastroenterology 2004; 126: Suppl. 2, A112.

15 Marinaki AM, Ansari A, Duley JA, et al. Adverse drug reactions to azathioprine therapy are associated with polymorphism in the gene encoding inosine triphosphate pyrophosphatase (ITPase). Pharmacogenetics 2004; 14: 181-187.

16 Fabre MA, Jones DC, Bunce M, et al. The impact of thiopurine s-methyltransferase polymorphisms on azathioprine dose 1 year after renal transplantation. Transpl Int 2004; 17: 531-539.
17 Anstey AV, Wakelin S, Reynolds NJ, British Association of Dermatologists Therapy, Guidelines and Audit Subcommittee. Guidelines for prescribing azathioprine in dermatology. Br J Dermatol 2004; 151: 1123-1132.

18 Van Aken J, Schmedders M, Feurerstein G, Kollek R. Prospects and limits of pharmacogenetics: the thiopurine methyl transferase (TPMT) experience. Am J Pharmacogenomics 2003; 3: 149-155.

19 Schwab M, Schaffeler E, Marx C, et al. Azathioprine therapy and adverse drug reactions in patients with inflammatory bowel disease: impact of thiopurine Smethyltransferase polymorphisms. Pharmacogenetics 2002; 12: 421-423.

20 Marra CA, Esdaile JM, Anis AH. Practical Pharmacogenetics: The cost effectiveness of screening for thiopurine $S$-methyltransferase polymorphisms in patients with rheumatological conditions treated with azathioprine. J Rheumatol 2002; 29: 2507-2512.

21 Winter J, Walker A, Shapiro D, Gaffney D, Spooner RJ, Mills PR. Cost-effectiveness of thiopurine methyltransferase genotype screening in patients about to commence azathioprine therapy for treatment of inflammatory bowel disease. Aliment Pharmacol Ther 2004; 20: 593-599.

22 Priest VL, Begg EJ, Gardiner SJ, et al. Pharmacoeconomic analyses of azathioprine, methotrexate and prospective pharmacogenetic testing for the management of inflammatory bowel disease. Pharmacoeconomics 2006; 24: 767-781.

23 van den Akker-van Marle ME, Gurwitz D, Detmar SB, et al. Cost-effectiveness of pharmacogenomics in clinical practice: a case study of thiopurine methyltransferase genotyping in acute lymphoblastic leukemia in Europe. Pharmacogenomics 2006; 7: 783-792.

24 Tavadia SM, Mydlarski PR, Reis MD, et al. Screening for azathioprine toxicity: a pharmacoeconomic analysis based on a target case. J Am Acad Dermatol 2000; 42: 628-632.

25 Gisbert JP, Luna M, Mate J, Gonzalez-Guijarro L, Cara C, Pajares JM. Choice of azathioprine or 6-mercaptopurine dose based on thiopurine methyltransferase (TPMT) activity to avoid myelosuppression. A prospective study. Hepatogastroenterology 2006; 53: 399-404.

26 Sayani FA, Prosser C, Bailey RJ, Jacobs P, Fedorak RN. Thiopurine methyltransferase enzyme activity determination before treatment of inflammatory bowel disease with azathioprine: effect on cost and adverse events. Can J Gastroenterol 2005; 19: 147-151.

27 Carter MJ, Lobo AJ, Travis SPL. Guidelines for the management of inflammatory bowel disease in adults. Gut 2004; 53: V1-V16.

28 Kornbluth A, Sachar DB. Ulcerative colitis practice guidelines in adults (update): American College of Gastroenterology, Practice Parameters Committee. Am J Gastroenterol 2004; 99: 1371-1385.

29 Luppi F, Cerri S, Beghe B, Fabbri LM, Richeldi L. Corticosteroid and immunomodulatory agents in idiopathic pulmonary fibrosis. Respir Med 2004; 98: 1035-1044. 